\title{
High-resolution TEM Analysis of Defect Structures in Mechanically Milled, Nanocrystalline Fe
}

\author{
M. MURAYAMA, J. M. HOWE, ${ }^{1)}$ H. HIDAKA ${ }^{2)}$ and S. TAKAKI ${ }^{21}$ \\ Physical Metallurgy Group, Steel Research Center, National Institute for Materials Science, Tsukuba 305-0047 Japan. \\ 1) Department of Materials Science and Engineering, University of Virginia, Charlottesville, VA 22904 USA. \\ 2) Department of Materials Science and Engineering, Kyushu University, Fukuoka 812-8581 Japan.
}

(Received on May 24, 2002; accepted in final form on December 6, 2002)

\begin{abstract}
Grain interior and grain-boundary structures of mechanically milled ultrafine-grained iron powder were investigated with atomic resolution using high-resolution transmission electron microscopy (HRTEM). Grain boundaries in the powder appear wavy and irregular, with no amorphous or other unusual defect structures present. Diffraction analysis of HRTEM images indicates that elongated grains approximately $100 \mathrm{~nm}$ long and $20 \mathrm{~nm}$ wide consist of several sub-grains along their length, each rotated by several degrees around a common $\langle 110\rangle$ axis. The presence of partial disclination dipoles in the sub-grains was also observed by HRTEM. Such structures indicate that shear, fragmentation and rotation of grains several to tens of nanometers in diameter occurs during severe plastic deformation. A mechanism for the formation of an ultrafine-grained structure in mechanically milled Fe involving partial disclinations is proposed.
\end{abstract}

KEY WORDS: high-resolution transmission electron microscopy; nanostructure; ultrafine-grained material; mechanically milled iron.

\section{Introduction}

Ultrafine-grained metallic materials tend to have high strength without sacrificing toughness and ductility; thus, they have attracted considerable research interest as well as industrial attention. It is well known that reduction of the grain size to the nanometer range can lead to remarkable mechanical behavior. For example, the hardness of finegrained iron alloys increases significantly with decreasing grain size as the average grain size becomes smaller than $1 \mu \mathrm{m}^{1-5)}$ Factors that have been considered to account for this behavior include the grain size, grain-boundary structure and internal defect structure. Gleiter ${ }^{6)}$ proposed that the structure of grain boundaries in ultrafine-grained materials is different from that of conventional materials, whereas other experimental results have shown that the structures are similar. ${ }^{7-9)}$ Previous high-resolution transmission electron microscopy (HRTEM) observations have revealed the presence of substantial local elastic strains in such materials, as evidenced by changes in image contrast, ${ }^{10,11)}$ as well as defect structures such as disclination dipoles. ${ }^{12}$ ) These features indicate that further detailed HRTEM may be beneficial in characterizing the nature of ultrafine-grained materials. The aim of this study was to reveal the grain structure and types of defects that are present in ultrafinegrained iron powder produced by mechanical milling. Severe plastic deformation (SPD) has recently become a popular processing method to produce ultrafine-grained $\mathrm{Fe}$ and other materials, ${ }^{13-23)}$ although the detailed mechanisms of grain refinement and the grain-refining strengthening are not clearly understood. HRTEM is particularly effective for characterizing these nanostructures, since it can reveal the atomic arrangements of both grain interiors and boundary regions in nano-particles and materials.

\section{Experimental}

A commercial pure iron powder $(\mathrm{Fe}-0.007 \mathrm{C}-0.03 \mathrm{Cr}-$ $0.04 \mathrm{Si}-0.24 \mathrm{Mn}$, wt $\%$ ) was mechanically milled with stainless steel balls under an Ar gas atmosphere in a planetary ball mill for up to $360 \mathrm{ks}$. After this fabrication process, the Vickers hardness of the mechanically milled iron (MM-Fe) powder reached 850 . The procedure of making the MM-Fe powder is described in detail elsewhere. ${ }^{21)}$

TEM specimens were prepared from as-milled Fe powder according to the following method. A small amount of MM-Fe powder was mounted in epoxy resin (PETROPOXY 154) and cured at $100^{\circ} \mathrm{C}$ for $30 \mathrm{~min}$. After mechanical grinding to about $0.05 \mathrm{~mm}$, the mounted specimen was glued onto a $\mathrm{Cu}-\mathrm{Be}$ alloy oval-hole grid and ion milled to perforation using a Gatan Duo-Mill Model 600 with a liquid-nitrogen cold stage. High-resolution TEM was performed in a JEOL JEM-4000EX microscope equipped with a UHP40H pole-piece and operating at $400 \mathrm{kV}$. The pointresolution of the microscope at Scherzer defocus $(-49.0$ $\mathrm{nm}$ ) is $0.18 \mathrm{~nm}$ and this is sufficient to image the atomic structure of Fe when it is oriented such that the $\langle 111\rangle$ axis is parallel to the viewing direction. ${ }^{12)}$ Subsequent image analysis was performed using Gatan DigitalMicrograph ${ }^{\mathrm{TM}}$ software on a Macintosh computer. 


\section{Results and Discussion}

A bright-field (BF) TEM image and corresponding selected-area diffraction pattern of the grain structure in the MM-Fe specimen are shown in Fig. 1. The grain structure appears to consist of either lamellae of relatively large elongated grains or smaller equiaxed grains separated by highangle boundaries. The large elongated grains appear to be a shear-band structure, ${ }^{24)}$ which is frequently observed in highly deformed metallic alloys. The presence of this shearband structure is also confirmed by the intensity variations

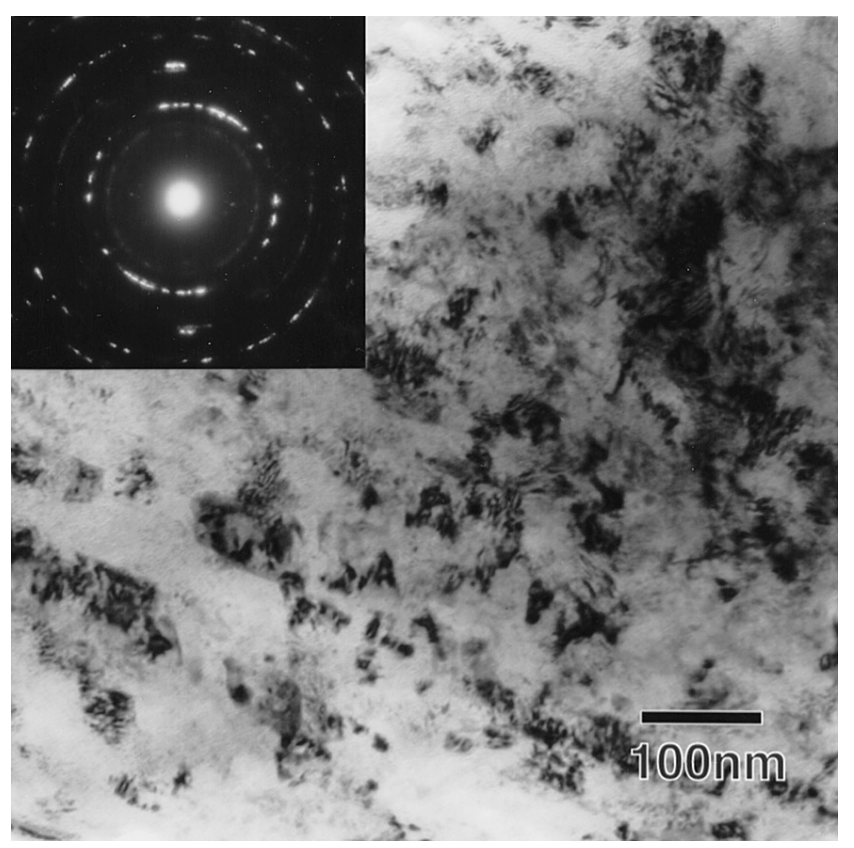

Fig. 1. Bright-field TEM image and corresponding selected-area diffraction pattern of the typical grain structure in the MM-Fe powder. in the diffraction rings, which are partly aligned rather than random around the rings (see inset selected-area diffraction pattern in Fig. 1). The sizes of individual grains in the shear bands ranged from approximately 20 to $100 \mathrm{~nm}$ in the longitudinal direction and their width appeared to be of the same order. Apart from contrast due to Moire effects in the image, the contrast in the interiors of the relatively larger grains is not uniform, suggesting that their internal structure varies. Similar observations have been reported in earlier work. ${ }^{1321)}$ In comparison, the size of the smaller equiaxed grains was around $20 \mathrm{~nm}$ and these tended to display fairly uniform contrast.

Figure 2(a) shows a HRTEM image taken from the area indicated by a box in the inset BF image in Fig. 2(b). Most of the grains in the image are in or near a $\langle 111\rangle$ orientation. The average size of the individual crystalline regions in Fig. 2 is close to that of the small grains shown in Fig. 1, i.e., approximately $20 \mathrm{~nm}$. Comparison of the BF and HRTEM images in Figs. 2(b) and 2(a), respectively, shows that what appear to be large grains in the BF image are actually composed of several smaller crystalline regions separated by low-angle boundaries, i.e., sub-grains, in the HRTEM image. In order to examine this effect more closely, the local orientation of individual crystalline regions in the HRTEM image was estimated by performing fast Fourier transforms (FFT's) of the regions using the FFT routine in DigitalMicrograph $^{\mathrm{TM}}$ software. Fourteen different areas (approximately $5 \times 5 \mathrm{~nm}$ square) were compared, as indicated by the locations of the numbers in Fig. 2(a). The FFT's from these regions are shown in Fig. 3. Area 7 in Figs. 2(a) and 3 is a region where the $\langle 111\rangle$ direction in a grain is almost parallel to the incident electron beam, thus leading to three clear sets of $\{110\}$ atom planes $(d=0.203 \mathrm{~nm})$ in the image (see further enlargement of HRTEM image in Fig. 4).

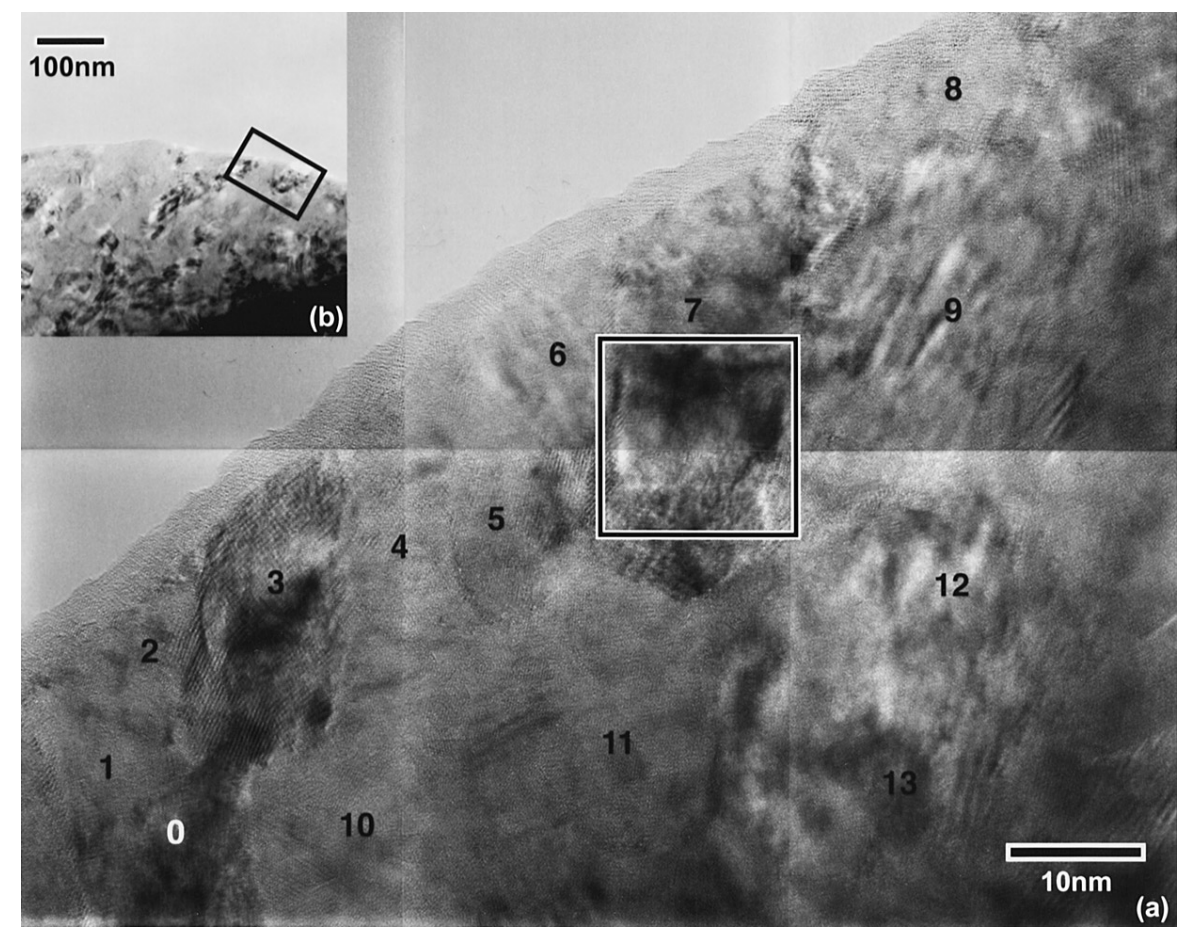

Fig. 2. (a) HRTEM image of the MM-Fe powder taken near a $\langle 111\rangle$ orientation and Scherzer defocus. (b) BF image with the area in Fig. 2(a) indicated by a box. 

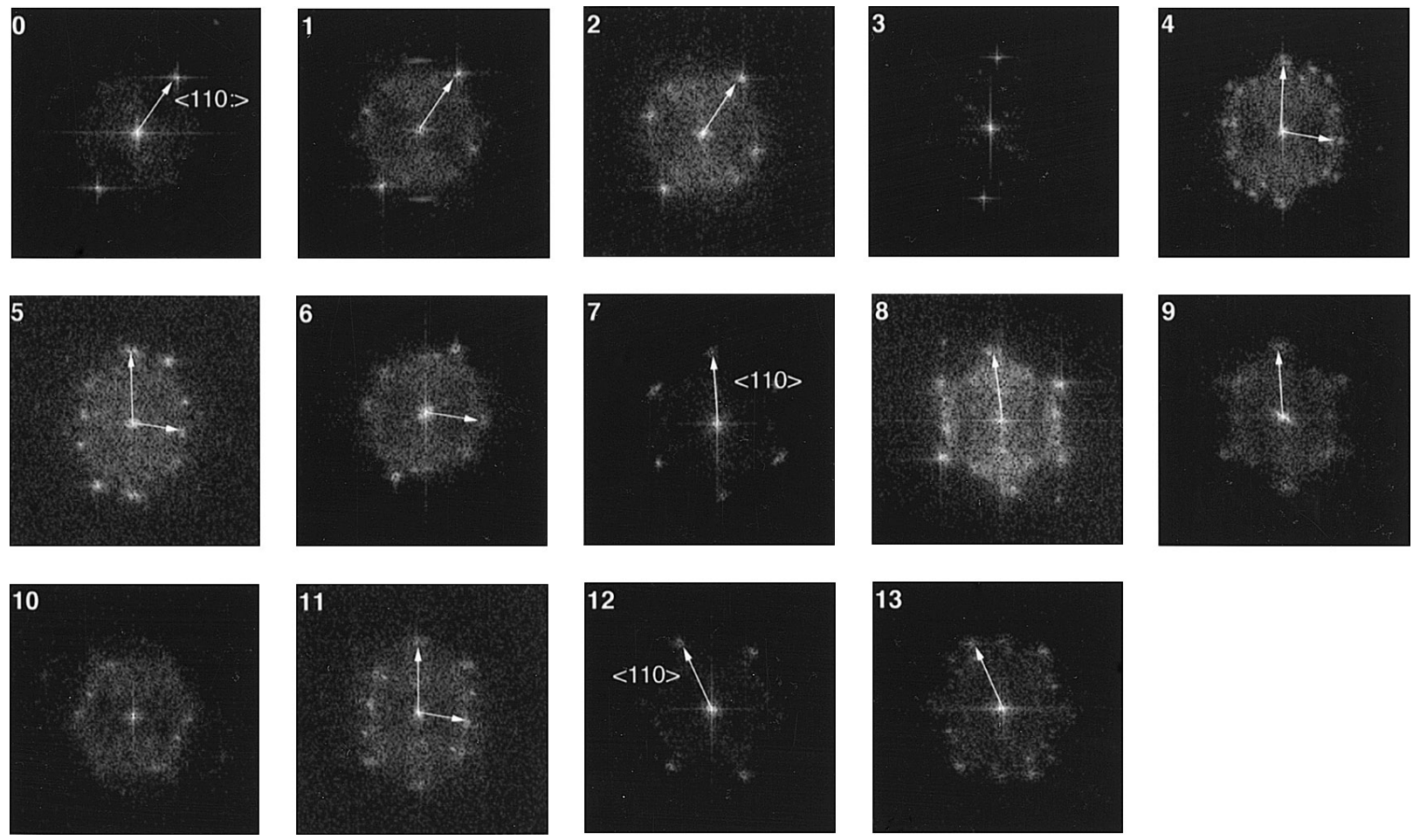

Fig. 3. Fast Fourier transforms (FFT's) taken from the grains numbered in Fig. 2(a), showing their relative crystal orientation. Arrows in the FFT's from grains $0,1,2$ and 7, 8 and 9 indicate a common $\langle 110\rangle$ rotation axis. Arrows in FFT's 4, 5, 6 and 11 indicate common spots.

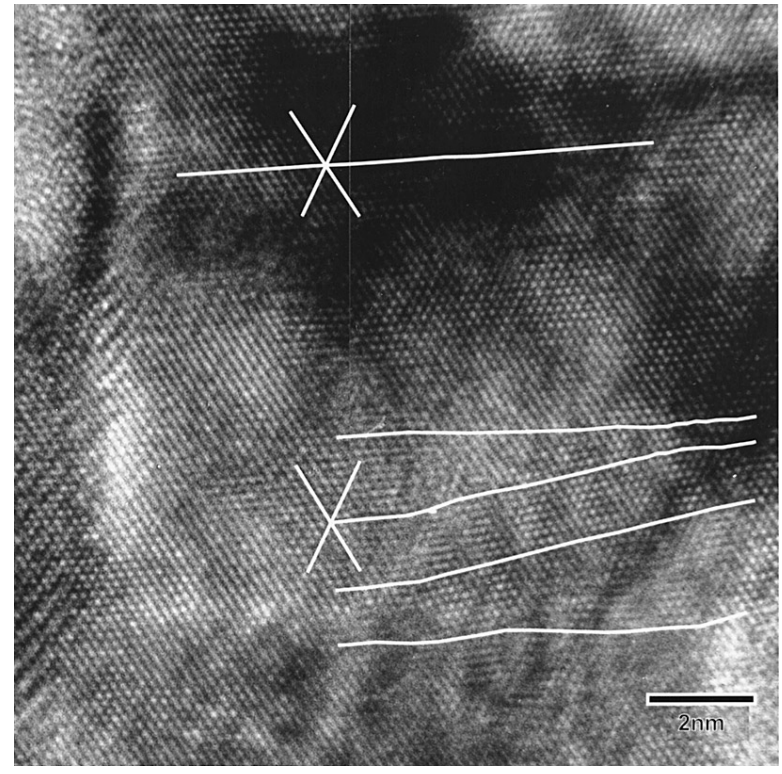

Fig. 4. A magnified HRTEM image of area 7 in Fig. 2(a). The grain is almost in an exact $\langle 111\rangle$ orientation and the hexagonal arrangement of white spots in the image corresponds to columns of Fe atoms in a $\langle 111\rangle$ crystal orientation. White lines are superimposed on the three sets of $\{110\}$ planes to highlight the distortion of the nearly horizontal set of $\{110\}$ planes in the region of the disclination dipole.

The relative rotations between adjacent grains in Fig. 2(a) can be examined by comparing their corresponding FFT's in Fig. 3. It is evident that grains that display similar contrast in Fig. 2 tend to be separated by low-angle boundaries, while those that display much different contrast are separated by high-angle boundaries. For example, areas 0,1 and 2 in Fig. 2(a) look similar and their corresponding FFT's in Fig. 3 have a common strong set of 110 reflections, indicated by the arrows in Fig. 3. Notice that these spots stay strong in the FFT's from areas 0,1 and 2 while the other reflections change in intensity. This feature indicates that the individual crystalline regions are aligned and rotating slightly about a common $\langle 110\rangle$ direction. There is a large change in contrast as one moves out of this band into region 3 in Fig. 2, and the corresponding FFT from region 3 in Fig. 3 shows a rotation between the 110 spots of about $28^{\circ}$, indicating that this is a high-angle boundary. The FFT's in Fig. 3 show that grains 7, 8 and 9 are similarly rotated about a common $\langle 110\rangle$ axis (arrows), as are regions 12 and 13 , although the angle between the common $\langle 110\rangle$ axes for these regions is about $23^{\circ}$, again indicating a high-angle boundary. Regions 4, 5, 6 and 11 also share common spots in Fig. 3 (arrows) and display similar contrast in Fig. 2. Thus, the results from these FFT's support the visual impression that the interiors of large grains in the shear band structure observed in typical BF TEM images consist of several smaller sub-grains, rotated about a common $\langle 110\rangle$ axis. The rotation angle between such sub-grains was typically less than about $7^{\circ}$, suggesting that rotation can occur gradually among small regions within a shear band during mechanical milling. It should be noted that FFT's taken from areas 4 and 5 display two sets of $\langle 110\rangle$ reflections at an angle of $30^{\circ}$. This indicates that the boundary between these two grains may be inclined to the electron beam so that the grains overlap through the foil thickness. Evidence of grain overlap can be seen in some of the other FFT's, but this feature does not affect the basic interpretation about the rotations between various grains from these patterns, particularly in cases such areas 0,1 and 2, which display mainly 
single-crystal patterns.

Some additional features observed in the HRTEM images are worthy of comment. First, the lattice fringes often appeared to bend locally in the images, indicating that the Fe lattice may be highly distorted. However, many nonuniform intensity changes occurred in the interiors of grains and across the images, as is clearly evident in Fig. 2(a). These effects are likely due to a combination of surface roughness, i.e., thickness variations in individual grains and along grain boundaries due to uneven thinning, the presence of an amorphous surface layer residual from ion milling, as well as the local orientation of and distortions in each grain, which have a large effect on electron diffraction and the resulting image contrast. These effects may cause the appearance of plane bending rather than real lattice distortions, so that such lattice distortions must be interpreted with caution.

Second, several grains appear mostly amorphous in Fig. $2(a)$, but display $\{110\}$ reflections in their diffraction patterns (FFT's) in Fig. 3. This indicates that these grains are tilted off a zone-axis orientation with planes that are diffracting too weakly to give rise to strong lattice fringes in the image, but that the planes are nevertheless still present. This image feature does not indicate the occurrence of amorphitization in the material. In fact, similar image characteristics have been observed for fragmented $\theta^{\prime}$ precipitates in SPD Al-Cu alloy, ${ }^{25)}$ where parts of precipitates that were rotated off a zone-axis imaging condition by deformation exhibited amorphous-like contrast.

Third, the morphology of the grain boundaries was highly irregular, as evident in Fig. 2. A few boundaries were somewhat planar, but most were quite curved and bumpy. These morphological features of the boundaries are similar to those found in SPD $\mathrm{Cu}$ and $\mathrm{Ni}^{2}{ }^{26)}$ Thus, it appears that this may be a common feature in heavily deformed metallic structures regardless of the metal or crystal structure. This is different from coarse-grained metallic alloys, where grain boundaries tend to be relatively planar and periodic. In contrast to some previous investigations, there was no evidence of amorphous or other types of unusual grain-boundary structures in the MM-Fe powder. While segments of some grain boundaries appeared amorphous-like, careful inspection of the images and/or FFT's of the regions always revealed the presence of weak lattice fringes, indicating that all of the boundaries were crystalline and similar to usual grain boundaries. The nearly amorphous contrast was attributed to fators such as surface roughness, etc., as discussed previously.

Lastly, Moiré fringes were frequently observed at inclined high-angle grain boundaries, whereas most lowangle boundaries provided faint images without accompanying Moire fringes. Assuming that the main component of the Moiré fringes is rotational, the period of the Moiré fringes $T$ is given by:

$$
T=\frac{d_{(110) \alpha}}{2 \sin \left(\frac{\beta}{2}\right)},
$$

where $d$ is the interplanar spacing of the $\{110\}$ planes in $\mathrm{Fe}$ and $\beta$ is the angle between the two grains. In the regions between 2 and 3, and between 6 and 7 in Fig. 2(a), $T$ is approximately $0.49 \mathrm{~nm}$, which indicates a rotation angle $\beta \approx 24.1^{\circ}$. This is reasonably close to the angles obtained from the FFT analysis in Fig. 3. It is worth noting that some spots with slightly smaller reciprocal spacings than the $\{110\}$ planes in the FFT's are evident in Fig. 3. The origin of these spots is not fully understood, but they may relate to the average projections of the atoms in individual grains tilted off their zone axis rather than the actual atom planes. ${ }^{27)}$

Figure 4 shows a magnified image of area 7, which is almost in an exact $\langle 111\rangle$ orientation. The grain can be divided into two parts following the changes in image contrast. In the upper portion, which is strongly diffracting and appears dark, the hexagonal pattern of the columns of Fe atoms are visible as white spots. All three sets of edge-on $\{110\}$ planes are almost straight and periodic. On the other hand, in the bottom part of the image, the set of $\{110\}$ planes that is approximately horizontal in the figure bends significantly; although the two sets of $\{110\}$ planes running vertically stay straight. In this region, the presence of two wedgeshaped regions facing one another indicates the presence of a partial disclination dipole in the grain. ${ }^{12)}$ The partial disclination dipole in Fig. 4 appears to consist of wedge disclinations with a Frank vector $\mathbf{w}$ parallel to the defect line, which corresponds to the $\langle 111\rangle$ viewing direction. It should be noted that determination of the wedge and twist components of the partial disclinatons cannot be made, because displacements parallel to the electron beam are not visible in the HRTEM image. Each wedge-shaped region has a number of terminating $\{110\}$ planes, which are individual dislocations with a Burgers vector of $\mathbf{b}=a / 2\langle 111\rangle$. The arrays of terminating $\{110\}$ planes can also be considered terminating tilt grain boundaries. ${ }^{28,29}$ Compared with complete tilt grain boundaries, termination tilt grain boundaries contain missing dislocations, and these are replaced by rotational elastic deformation in the crystal. ${ }^{30)}$

It is not possible to determine exactly how the partial disclination dipole in Fig. 4 formed, but it is possible to demonstrate how such dipoles can facilitate the shearing, fragmentation and rotation of metals in response to a high shear stress. A partial disclination dipole in Fe, such as that in Fig. 4, can be produced by the nucleation and arrangement of shear dislocations loops into arrays, with the dislocations stacked vertically above (or below) one another, as illustrated in Fig. 5. The loops can form under the application of a high shear stress $(\tau)$ in the directions indicated by arrows in Fig. 5(a) and glide apart, producing a shear dislocation dipole (with terminating planes marked by the symbol $\perp$ ). If the loop becomes sessile due to interaction with other existing defects in the material, repetition of this process on parallel slip planes under a sustained shear stress (Figs. 5(b)-5(c)) eventually produces an array of dislocation dipoles, collectively called a disclination dipole (or two terminating tilt grain boundaries of opposite sign). The crystal planes between the disclination dipole are sheared and rotated with respect to the surrounding material, as evident in the figure, with the magnitude of the shearing depending on the spacing among the dislocations in the arrays. The resulting dislocation configuration shown schematically on the right in Fig. 5(c) closely resembles the disloca- 


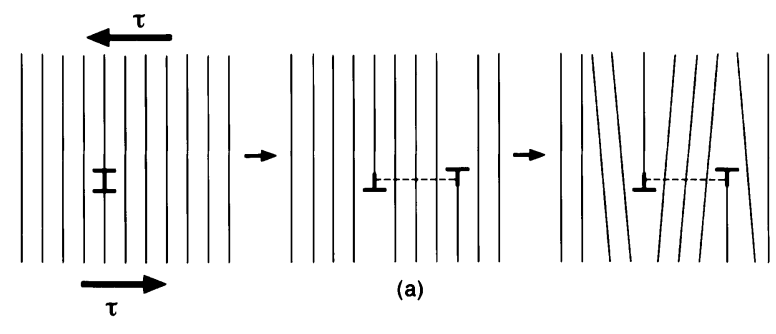

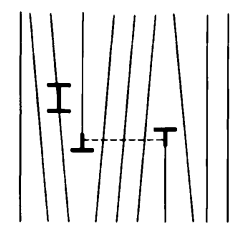
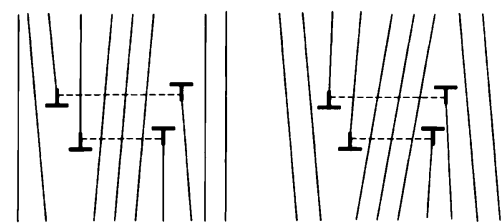

(b)
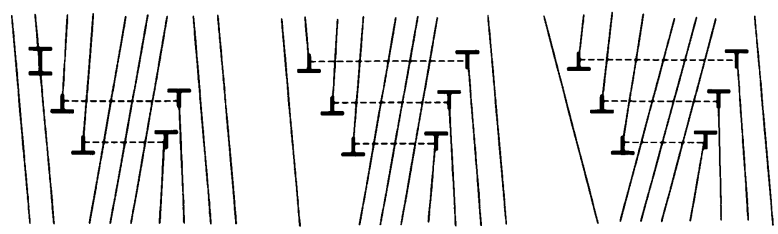

(c)

Fig. 5. (a)-(c) Illustrations showing how the generation of dislocation dipole loops (for example, with $\mathbf{b}=a / 2\langle 111\rangle$ ) one above the other under the application of a shear stress $(\tau)$, produces a partial wedge disclination dipole with crystal shearing and rotation in between.

tion configuration observed experimentally in Fig. 4. It should be noted that a variety of different mechanisms have been proposed to explain the nucleation of dislocations during deformation as well as the formation of boundary fragments by dislocation pile-up. ${ }^{30)}$ Most of mechanisms require the presence of obstacles, such as a cell wall, LomerCottrell barrier or a grain boundary, in order to lead to pile up of dislocations to form disclinations. The lower partial disclination in Fig. 4 is located in the corner of a sub-grain near a boundary, indicating that it may have resulted from pile-up at the boundary, or possibly emanated from the boundary during deformation. (A number of isolated dislocations (or terminating $\{110\}$ planes) were evident in subgrains in the HRTEM images, indicating that lattice dislocations may be available to form boundary fragments by pile-up.) This provides some evidence that the formation of disclination dipoles may be related to the presence of other defects such as grain boundaries in the material. Unfortunately, it is difficult to understand the formation of these defects from static HRTEM images, suggesting that the further in-situ HRTEM deformation studies may be necessary to clarify the mechanisms of partial disclination formation in metallic alloys.

The formation and migration of partial disclinations allows crystalline solids to shear and progressively rotate. The partial disclination dipoles in Fig. 4 are only about 3.5 $\mathrm{nm}$ apart, indicating that they allow volumes of material to shear and rotate at this size scale. Many such partial disclination dipoles were observed in the MM-Fe powder, as was the progressive rotation of small crystalline regions about a common $\langle 110\rangle$ crystallographic axis, as mentioned previously with regard to Fig. 3. Such features indicate that partial disclination dipoles facilitate the rotation and fragmen- tation of grains in shear bands under the applied shear strains introduced during mechanical milling. In fact, the crystal rotation produced by the partial disclination dipole in Fig. 4 is evident from the bending of the $\{110\}$ planes between them by about $9^{\circ}$ relative to the $\{110\}$ planes in the top and bottom parts of the grain. Hence, a mechanism for the formation of ultra-fine grained structures based on the reorientation process of individual grains can be proposed as follows. Defects such as partial disclinations form in grains or at grain boundaries, by rearrangement of individual dislocations into arrays or when the shear stress exceeds a critical level locally and arrays are generated, as illustrated in Fig. 5. Formation of the partial disclinations in the grains allows regions a few nanometers or larger in size to rotate and rearrange until they eventually combine and establish new low-angle grain boundaries. The misorientation of these boundaries continues to increase with additional deformation until additional grains separated by high-angle boundaries are formed. This process continues until an ultrafine-grain structure is achieved. The formation and stability of defects such as partial disclination dipoles depends on the grain size of a material ${ }^{31)}$ and may be favored at small grain sizes, although this factor remains to be determined.

\section{Acknowledgments}

We acknowledge the helpful comments of Y. Kimura and W. T. Reynolds, Jr. on this work. Support by the Ministry of Education, Culture, Sports, Science and Technology of Japan (M.M.) and by the National Science Foundation, Division of Materials Research Grant DMR-9908855 (J.M.H. and M.M.) is gratefully acknowledged.

\section{REFERENCES}

1) J. S. C. Jang and C. C. Koch: Scr. Metall. Mater, 24 (1990), 1599.

2) T. G. Nieh and J. Wadsworth: Scr. Metall. Mater., 15 (1991), 955.

3) L. He and E. Ma: Nanostructured Mater., 7 (1996), 327.

4) M. Zhu, J. H. Ahn, X. Z. Che, B. L. Li and Z. X. Li: J. Mater. Sci. Lett., 17 (1998), 445.

5) J. E. Carsley, A. Fisher, W. W. Milligan and E. C. Aifantis: Metall. Mater. Trans. A, 29A (1998), 2261.

6) H. Gleiter: Prog. Mater. Sci., 33 (1989), 233.

7) G. Thomas, R. W. Siegel and J. A. Eastman: Scr. Metall. Mater, 24 (1990), 201.

8) R. W. Siegel and G. J. Thomas: Ultramicroscopy, 40 (1992), 376.

9) E. A. Stern, R. W. Siegel, M. Newville, P. G. Sanders and D. Haskel: Phys. Rev. Lett., 75 (1995), 3874.

10) X. Zhu, R. Birringer, U. Herr and H. Gleiter: Phys. Rev. B, 35 (1987), 9085.

11) W. Wunderlich, Y. Ishida and R. Maurer: Scr. Metall. Mater, 24 (1990), 403.

12) M. Murayama, H. Hidaka, S. Takaki and J. M. Howe: Science, 295 (2002), 2433.

13) H. Shen, Z. Li, B. Günther, A. V. Korznikov and R. Z. Valiev: Nanostructured Mater, 6 (1995), 385.

14) V. A. Teplov, V. P. Pillugin, V. S. Gaviko and E. G. Chernyshov: Nanostructured Mater, 6 (1995), 437.

15) V. V. Stolyarov, V. V. Latysh, V. A. Shundalov, D. A. Salimonenko, R. K. Islamgaliev and R.Z. Valiev: Mater. Sci. Eng., A234/236 (1997), 339.

16) S. Ferrasse, V. M. Segal, K. T. Hartwig and R. E. Goforth: Metall. Mater. Trans. A, 28A (1997), 1047.

17) Y. Iwahashi, Z. Horita, M. Nemoto and T. G. Langdon: Acta Mater., 9 (1998), 3317.

18) O. N. Senkov, F. H. Froes, V. V. Stolyrov, R. Z. Valiev and J. Liu: Nanostructured Mater, 10 (1998), 691. 
19) P. J. Hurley, P. D. Hodgson and B. C. Muddle: Scr. Mater., 40 (1999), 433.

20) V. M. Segal, V. I. Reznikov, A. E. Drobyshevskiy and V. I. Kopylov: Russ. Metall., 1 (1981), 99.

21) Y. Kimura, H. Hidaka and S. Takaki: Mater. Trans., JIM, 40 (1999), 1149.

22) H. J. Fecht, E. Hellstern and W. L. Johnson: Metall. Trans., 21A (1990), 2333.

23) M. Umemoto, Z. G. Liu, K. Masuyama, X. J. Hao and K. Tsuchiya: Scr. Mater, 44 (2001), 1741.

24) J. Hirsch, K. Lucke and M. Hatherky: Acta Metall., 36 (1988), 2905.
25) M. Murayama and K. Hono: Acta Mater, 49 (2001), 21.

26) Z. Horita, D. J. Smith, M. Nemoto, R. Z. Valiev and T. G. Langdon: J. Mater. Res., 13 (1998), 446.

27) J.-O. Malm and M. A. O'Keefe: Ultramicroscopy, 68 (1997), 12.

28) A. E. Romanov and V. I. Vladimirov: Dislocations in Solids, Vol. 9, ed. by F. R. N. Nabarro, North-Holland, Amsterdam, (1992), 191.

29) J. Michler, Y. von Kaenel, J. Stiegler and E. Blank: J. Appl. Phys., 83 (1998), 187.

30) M. Seefeldt: Rev. Adv. Mater. Sci., 2 (2001), 44.

31) V. G. Gryaznov, A. M. Kaprelov and A. E. Romanov: Scr. Metall., 23 (1989), 1443. 